Meta

Journal des traducteurs

Translators' Journal

\title{
LADMIRAL, Jean-René (2014) : Sourcier ou cibliste. Paris : Belles \\ Lettres, 303 p.
}

\section{Christine Pagnoulle}

Volume 62, numéro 3, décembre 2017

URI : https://id.erudit.org/iderudit/1043955ar

DOI : https://doi.org/10.7202/1043955ar

Aller au sommaire du numéro

\section{Éditeur(s)}

Les Presses de l’Université de Montréal

\section{ISSN}

0026-0452 (imprimé)

1492-1421 (numérique)

Découvrir la revue

Citer ce compte rendu

Pagnoulle, C. (2017). Compte rendu de [LADMIRAL, Jean-René (2014) : Sourcier ou cibliste. Paris : Belles Lettres, 303 p.] Meta, 62(3), 647-648.

https://doi.org/10.7202/1043955ar

Ce document est protégé par la loi sur le droit d'auteur. L'utilisation des services d'Érudit (y compris la reproduction) est assujettie à sa politique d'utilisation que vous pouvez consulter en ligne.

https://apropos.erudit.org/fr/usagers/politique-dutilisation/
Cet article est diffusé et préservé par Érudit.

Érudit est un consortium interuniversitaire sans but lucratif composé de l’Université de Montréal, l’Université Laval et l'Université du Québec à Montréal. Il a pour mission la promotion et la valorisation de la recherche. https://www.erudit.org/fr/ 


\section{DOCUMENTATION}

LAdMiRAL, Jean-René (2014): Sourcier ou cibliste. Paris: Belles Lettres, 303 p.

\section{La traduction comme quête d'idiomaticité}

Philosophe, traducteur, enseignant, chercheur, Ladmiral est une figure marquante dans le monde des études en traduction. Il faut dire qu'il publiait déjà dans ce domaine dès les années 1970, notamment son ouvrage de référence Traduire: théorèmes pour la traduction, à une époque où l'intérêt pour le sujet était encore limité. Depuis, non seulement il a poursuivi ses travaux de traduction (essentiellement des philosophes allemands), mais il a également continué à enseigner et à faire de la recherche (à la retraite, il est toujours très actif, entre autres au sein du CRATIL, Centre de recherche appliquée sur la traduction, l'interprétation et le Langage). Il passe de colloque en colloque avec le même souci de faire bien plus qu'une intervention: il suit toutes les communications et ne manque pas de donner son avis, avec autant de courtoisie que de conviction, dans un français vieille France dont le caractère châtié frappe encore davantage à l'oral qu'à l'écrit.

Sourcier ou cibliste est un recueil d'une cinquantaine d'articles et conférences. Comme l'indique le titre, ils tournent tous autour de l'opposition entre une traduction littéraliste et une traduction «idiomatique», c'est-à-dire qui recrée le texte dans le propre de la langue cible. Déjà Cicéron distinguait la traduction servile de la traduction brillante, qui n'hésite pas à s'écarter de la lettre pour faire chatoyer l'esprit.

Mais à quoi correspond cet antagonisme? La tendance à opposer lettre et esprit se comprend aisément: le texte est fait de mots, de signifiants - c'est la lettre. Pourtant ce qu'il exprime n'existe que par ses mots. L'esprit est dans la lettre, et la lettre est esprit, pourrions-nous dire. Dans l'acte de traduire (magie? alchimie? même pas! juste savoir-faire), les mots changent, de même que leur disposition et leurs échos. Cependant, dans le texte cible, c'est encore bel et bien avec la lettre qu'il faut façonner l'esprit.

Autre indication présente dans le titre: l'exclusive. Impossible, affirme Ladmiral d'être à la fois sourcier et cibliste, pour reprendre les termes qu'il avait lancés dans le feu de la discussion à Londres en 1983 et étayés dans un article de la Revue d'esthétique en 1986. Il se place ainsi dans le registre de la polémique, de la confrontation. Une manie française? Pour illustrer la tendance «sourcière», Ladmiral mentionne deux grands théoriciens de la traduction, qui étaient aussi de grands traducteurs ( amis personnels et adversaires théoriques»): Henri Meschonnic et Antoine Berman. Est-ce bien légitime? Allant à l'encontre des traductions de la Bible qui nous sont familières, Meschonnic nous rappelle la respiration du verset hébreu et propose des traductions qui font entendre ce souffle. Rien à voir avec la bible de Segond ou celle du roi Jacques, mais qui peut dire que ce n'est pas là l'effet même, poétique, quasi hypnotique, du texte original? Dans son commentaire de diverses traductions d'une ode de John Donne, Berman (1995) met en évidence l'excellence de la traduction/ adaptation d'Octavio Paz, un poème qui retrouve/ recrée la légèreté badine de ce texte érotique, sans se préoccuper de respecter la forme (les vers de Paz sont plus courts, tout le poème s'est condensé). Ceci pour dire que les traductions que Ladmiral réfute, à juste titre, comme "sourcières" devraient sans doute être appelées "ultra-sourcières", de même que notre auteur parle de traductions « ultraciblistes» lorsqu'elles s'éloignent du texte source sans jamais y revenir. S'il y a opposition dichotomique, c'est entre ces deux caricatures. Notons d'ailleurs que les deux mouvements décrits par Schleiermacher, la traduction qui amène le lecteur à l'auteur et celle qui amène l'auteur au lecteur, sont en fait complémentaires: le traducteur ne peut que faire les deux. C'est en rendant le texte source «idiomatique» dans la langue cible que le lecteur pourra découvrir l'auteur dans sa singularité.

La réflexion de Ladmiral est jalonnée de déclarations qui sont tout simplement l'expression du bon sens. Il nous dit, par exemple, que la traduction est à proprement parler l'interface ou l'«interphase» entre la lecture-interprétation et la reformulation en langue cible (p. 169-70). Ce qu'il met en cause, c'est le souci de coller au texte source, qui va produire un texte obscur, parfois quasi illisible, là où l'original est clair. Quiconque a enseigné la traduction reconnaît là le réflexe quasi désespéré de la plupart des étudiants qui se cramponnent à une notion dévoyée de fidélité. Or ce type de traduction, loin de servir l'auteur, non seulement malmène la langue cible mais déforme le message: «sous couvert de fidélité apparente [une traduction qui est en fait un transcodage mot à mot], le traducteur [se montre] deux fois traître: traître à la langue cible, à la langue d'arrivée dans laquelle il traduit, et traître envers son texte source, envers le texte d'origine» (p. 188). L'écart pris par rapport au texte afin de mieux y revenir est un des principes les plus difficiles à faire accepter. 
Parfois, en plus, il faut non seulement s'éloigner du texte mais aussi l'élaguer pour lui donner plus de chances de s'épanouir.

Ladmiral évoque ici le concept de dissimilation: «La traduction doit toujours s'éloigner de l'original, en prenant appui sur lui, pour lui donner rendez-vous au plus près» (p. 199). L’analogie qui suit (la figure de rock ' $n$ roll où les corps s'éloignent, se repoussent, pour mieux se retrouver), d'autant plus séduisante qu'elle en appelle au plaisir physique, fait voir les deux temps de l'éloignement et du rapprochement, certes, mais je ne crois pas qu'elle éclaire vraiment le processus en œuvre dans la traduction. Je préfère la formulation non métaphorique (ou si peu) de l'énoncé suivant: "paradoxalement, c'est dans le même mouvement contradictoire que j'épouse au plus près l'esprit du texte source dans le moment même où je le rends (dans la langue cible) en l'éloignant résolument de la lettre de sa textualité» (p. 96).

$\mathrm{Si}$ je souscris pleinement à ces commentaires, d'autres notions me laissent sceptique. Ainsi le "salto mortale de la déverbalisation", cher à l'école de Paris et mentionné à maintes reprises dans ce recueil. Là, rien à faire, je n'arrive pas à comprendre; dans mon expérience de la traduction, il y a toujours des mots, même s'il m'arrive de «schtroumpfer» à tout va, faute de disposer immédiatement du mot, de la tournure correcte en langue cible. Autre réticence: juste après avoir donné la définition de la traduction citée plus haut (interface ou interphase), Ladmiral laisse entendre que c'est uniquement lorsque la traduction se présente comme simple transcodage (ce qui est rare) qu'elle se fait sans labeur, sans « travail intellectuel et psychologique» (p. 170). Or je tiens au contraire que parfois ce sont de véritables nœuds dans la traduction qui peuvent se dénouer d'eux-mêmes, des tournures difficiles qui se résolvent, simplement... en ne cherchant pas. C'est là le miracle de la disponibilité d'esprit dont parle Douglas Robinson (1997).

Le volume se termine par un parallèle entre théologie et traduction. Il y a tout d'abord cette propension à sacraliser le texte source, plus encore s'il s'agit d'un texte sacré, qui devient dès lors quasi intouchable. Pour le Coran, la cause est entendue: il ne peut être traduit. Il en allait de même pour l'Ancienne Alliance, la Bible judaïque, et seul le miracle des Septante a rendu acceptable la version grecque d'Alexandrie. D'où la tentation d'un littéralisme poétique et la beauté obscure de la traduction d'un Chouraqui. Mais la traduction même est «une opération d'ordre théologique» (p. 279) puisqu'elle nous donne accès à ce qui est caché. Elle révèle, nous dit Ladmiral, l'impensé théologique qui expliquerait la passion des querelles entre spécialistes. Par ailleurs, dans sa fonction révélatrice, elle est "médiatrice» comme la mère de Jésus? Ou comme l'interprète de liaison au tribunal? Je ne suis pas sûre que cette suggestion, qui peut sembler quasi blasphématoire, soit une sécularisation abusive: le traducteur est aussi et avant tout "truchement», celui par qui advient le contact, et tout à la fois une certaine compréhension et toujours aussi irrépressiblement, l'incompréhension.

Christine Pagnoulle Université de Liège, Liège, Belgique

\section{RÉFÉRENCES}

Berman, Antoine (1995): Pour une critique des traductions: John Donne. Paris: Gallimard.

Robinson, Douglas (1997): Becoming a Translator. Londres/New York: Routledge.

Biel, Eucja (2014): Lost in the Eurofog: The Textual Fit of Translated Law. Frankfurt: Peter Lang, 347 p.

Let me start with a sporting analogy. Every two years Ireland and Australia compete in a football match using a set of compromise rules agreed by the Gaelic Athletic Association and the Australian Football League. The resulting sport is a hybrid game containing elements of both national sports (the Australians for example must come to terms with a round ball and the Irish have to adapt to the more robust tackle that is a feature of the Australian game). The making of EU law is also all about compromise. Legal texts are kicked around from one institution to another and are subject to various linguistic inputs which make it impossible to determine what the original text is. Bengoetxea describes the process thus:

According to the Community method for elaborating law, the Commission proposes and the Council and Parliament dispose. It might be the case that the preparatory materials leading to and arising from negotiations carried out by the legislators at the Commission, the Committees of national experts, the Council (and the Committee of Permanent Representatives - COREPER) and the Parliament committees and Plenum, and even the input of the Committee of Regions or the European Economic and Social Committee are themselves the result of the combination of translations going in different directions. By contrast with many policy and consultation documents (for example, Green and White Papers) available normally in English or French, perhaps also occasionally in German, Spanish or Dutch, a 